\title{
Perfil neuropsiquiátrico na doença de Alzheimer e na demência mista
}

\author{
Neuropsychiatric profile in Alzheimer's disease and in mixed dementia \\ Simone Rios Fonseca', Ana Paula Borges Santos', Mariana Alves de Almeida', Henrique Cerqueira \\ Guimarães', Thiago André Fuscaldi Corrêa', Rogério Gomes Beato', Paulo Caramelli
}

\section{RESUMO}

Alguns estudos sugerem que infartos cerebrais possam agravar a demência em pacientes com doença de Alzheimer (DA) e que sintomas neuropsiquiátricos sejam comuns tanto na DA quanto na demência vascular (DV). Doença cerebrovascular concomitante à DA incorre na chamada demência mista (DM). Objetivos: Comparar a freqüência e o perfil dos sintomas neuropsiquiátricos em uma amostra de pacientes com DA e DM. Métodos: Análise retrospectiva dos prontuários de 70 pacientes com diagnóstico de DA provável e 14 com DM. Informações sobre sintomatologia neuropsiquiátrica foram obtidas por meio dos relatos de familiares e cuidadores. Resultados: A média etária foi de 74,5 anos na DA e 75,1 na DM. 0 sintoma mais comum na DA foi agitação $(61,4 \%)$, enquanto na DM foi apatia $(71,7 \%)$. Na DM, nove $(64,3 \%)$ pacientes apresentavam $\geq 5$ sintomas, enquanto na DA, 40 (57,1\%) apresentavam $\leq 4$. Quarenta e cinco $(64,3 \%)$ pacientes com DA tinham $\geq 4$ anos de doença; na DM, 10 $(71,4 \%)$ tinham $\leq 3$ anos. Pacientes com DM mostraram menor duração de sintomas $(p<0,05)$, sugerindo que tenham procurado atendimento médico mais precocemente. Conclusões: Os pacientes com DM exibiram maior gravidade de sintomas neuropsiquiátricos, fato que pode ter sido responsável pela busca mais precoce de assistência especializada.

\section{ABSTRACT}

Some studies suggest that concomitant cerebral infarction may worsen the severity of dementia in patients with Alzheimer disease (AD) and that neuropsychiatric symptoms are common either in patients with $A D$ and vascular dementia. AD lesions together with cerebrovascular disease is commonly called mixed dementia (MD). Methods: A retrospective analysis was carried out in medical charts of 70 patients with probable AD and 14 with MD. Information on neuropsychiatric symptoms was based on caregivers' and families' reports. Results: Mean age was 74.5 years in $A D$ and 75.1 in MD. The most common symptom in AD was agitation (61.4\%), while in MD apathy was more common (71.7\%). In MD, 9 (64.3\%) patients had 5 or more symptoms, while in $A D, 40$ (57.1\%) had 4 or less. Forty-five (64.3\%) patients with AD had more than 4 years of disease; in MD, 10 (71.4\%) had less than 3 years. Patients with MD showed shorter duration of symptoms ( $p<0.05)$, suggesting that these patients search earlier for medical treatment. Conclusions: Patients with MD exhibited a greater severity of neuropsychiatric symptoms, which may have been responsible for the earlier need of specialized assistance.

1 Grupo de Pesquisa em Neurologia Cognitiva e do Comportamento do Departamento de Clínica Médica da Faculdade de Medicina da Universidade Federal de Minas Gerais (UFMG). 


\section{INTRODUÇÃO}

Demência constitui síndrome caracterizada por declínio de memória e de outras funções cognitivas, de intensidade suficiente para interferir de maneira significativa sobre o desempenho funcional do paciente ${ }^{1,2}$.

A doença de Alzheimer (DA) e a demência vascular (DV) são as duas formas mais freqüentes de demência na população idosa ${ }^{3}$. A DA é condição neurodegenerativa em que há acúmulo de placas amilóides extracelulares e emaranhados neurofibrilares intraneuronais, enquanto a DV ocorre secundariamente à doença cerebrovascular (DCV) isquêmica (na maioria dos casos) ou hemorrágica, ${ }^{1,2,4}$.

As lesões da DA e da DCV (especialmente as de natureza isquêmica) freqüentemente ocorrem em associação ${ }^{5,6}$. As lesões vasculares concomitantes em pacientes com DA podem acarretar aumento dos efeitos patológicos desta última, acentuando o comprometimento cognitivo e resultando expressão mais precoce da demência, conforme já salientado por alguns autores ${ }^{7,8}$. A coexistência de DA e DCV recebe comumente a terminologia de demência mista (DM) ${ }^{6}$.

Os sintomas comportamentais estão entre as principais causas de sobrecarga do cuidador do paciente com demência, relacionando-se a maiores níveis de estresse nestes cuidadores ${ }^{9,10}$, visto que sua importante prevalência e impacto já são reconhecidos na prática clínica"1. Muitos estudos têm demonstrado que sintomas neuropsiquiátricos são comuns tanto em pacientes com DA quanto naqueles com $\mathrm{DV}^{8,12,13}$. Um destes estudos ${ }^{13}$ mostrou que praticamente metade dos pacientes que recebeu o diagnóstico de demência em um serviço ambulatorial especializado apresentava morbidade psiquiátrica significativa. Outros dois trabalhos ${ }^{8,14} \mathrm{se}$ mostraram conflitantes em definir se sintomas neuropsiquiátricos são mais comuns em pacientes com DA ou naqueles com DV.

O objetivo do presente estudo foi comparar os sintomas e o tempo de doença em casos de DA e DM acompanhados em um ambulatório especializado.

\section{MÉTODOS}

Uma análise retrospectiva foi desenvolvida em prontuários de 198 pacientes acompanhados no Ambulatório de Neurologia Cognitiva do Hospital das Clínicas da Universidade Federal de Minas Gerais (UFMG). Foram incluídos na análise aqueles pacientes que apresentavam um mínimo de três consultas no Hospital das Clínicas da UFMG, visto que necessariamente duas delas deveriam ter sido realizadas no referido ambulatório. A escolha de prontuários foi feita de maneira aleatória entre todos os pacientes atendidos pela unidade entre o período de janeiro de 2003 a julho de
2007. A coleta de dados foi realizada no período de julho a dezembro de 2007. Este estudo foi devidamente aprovado pelo Comitê de Ética em Pesquisa da UFMG.

A história clínica, os exames físico e neurológico, os achados laboratoriais e de neuroimagem foram utilizados para definição diagnóstica. O diagnóstico de demência teve como base os critérios do DSM-IV da American Psychiatric Association ${ }^{15}$. O diagnóstico de DA teve como base os critérios do NINCDS-ADRDA para DA provável ${ }^{16}$, enquanto o de DM foi dado aos pacientes com DA provável que apresentavam DCV significativa aos exames de neuroimagem (tomografia computadorizada ou ressonância magnética), correspondendo aos critérios de DA com DCV do NINDS-AIREN ${ }^{17}$.

Dos 198 prontuários de pacientes analisados, 135 preenchiam critérios diagnósticos de demência. A DA foi responsável por $51,9 \%(n=70)$ destes casos e a DM, por $10,4 \%$ $(n=14)$ (Figura 1). Os restantes 51 (37,8\%) casos de demência estavam relacionados às seguintes etiologias: demência frontotemporal (16 casos; 11,8\%), DV (13 casos; 9,6\%), doença de Parkinson (quatro casos; 3\%), degeneração corticobasal (4 casos; 3\%), demência com corpos de Lewy (três casos; 2,2\%) e paralisia supranuclear progressiva (um caso; 0,7\%). Os dez $(7,4 \%)$ pacientes remanescentes não tinham ainda etiologia definida.

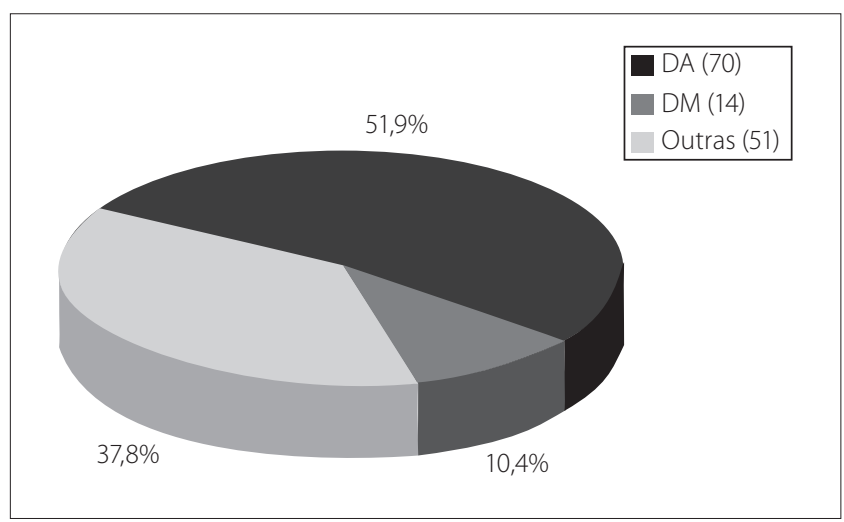

Figura 1. Freqüência de DA e DM no total de 135 casos de demência.

A caracterização dos sintomas neuropsiquiátricos apresentados pelos pacientes teve como base os relatos de seus familiares e cuidadores. Esses sintomas eram em número de 12: idéias delirantes, alucinações, apatia, ansiedade, humor deprimido, euforia, desinibição, irritabilidade, perambulação, alteração do sono/vigília e desordem alimentar. Os sintomas foram fundamentados nos itens do inventário neuropsiquiátrico ${ }^{18} \mathrm{e}$, apesar de não ter havido aplicação padronizada deste, tais sintomas eram questionados aos acompanhantes durante a consulta e, então, transcritos no prontuário.

O tempo de doença foi obtido somando-se o tempo de evolução relatado à primeira consulta com o tempo de 
acompanhamento do paciente no ambulatório. Os sintomas neuropsiquiátricos eram obtidos por meio de relatos em todo este tempo de acompanhamento. A escolha pela avaliação longitudinal teve como base a maior exuberância de dados que este tipo de metodologia proporciona.

Os dados obtidos dos prontuários foram transcritos para o programa EpiData e as análises estatísticas foram feitas por meio do programa Epilnfo e SPSS14, utilizando os testes t de Student e qui-quadrado.

\section{RESULTADOS}

O tempo médio de acompanhamento dos pacientes no Ambulatório de Neurologia Cognitiva foi de 15,7 \pm 12,9 meses. A média etária dos pacientes à primeira consulta foi de $74,5 \pm 7,2$ anos na DA e de 75,1 $\pm 7,6$ anos na DM. A pontuação média do primeiro mini-exame do estado mental (MEEM) realizado com os pacientes foi de 12,9 $\pm 6,9$ na DA e de 14,6 \pm 4,7 na DM. Não houve diferença estatisticamente significante entre os dois grupos, tanto em relação à média etária quanto em relação aos escores no primeiro MEEM, o que permitiu a comparação. Todos os pacientes com DM e 60 dos 70 pacientes com DA tinham dados do primeiro MEEM (Tabela 1).

O sintoma neuropsiquiátrico mais comum na DA foi agitação $(61,4 \%)$, enquanto na DM foi apatia (71,7\%). O sintoma menos observado, tanto entre os pacientes com DA quanto naqueles com DM, foi euforia, sendo verificado em 1,4\% dos pacientes com DA e em nenhum paciente com DM. Sete dos 12 sintomas foram encontrados mais em pacientes com

Tabela 1. Média etária e MEEM na DA e na DM.

\begin{tabular}{lccc}
\hline & DA & DM & $p$ \\
\hline Média etária & $74,5 \pm 7,2$ & $75,1 \pm 7,6$ & 0,7481 \\
MEEM & $12,9 \pm 6,9$ & $14,6 \pm 4,7$ & 0,2740 \\
\hline
\end{tabular}

DM do que naqueles com DA, quais sejam, idéias delirantes, alucinações, apatia, ansiedade, humor deprimido, irritabilidade e desordem alimentar.

Foram encontrados, ainda, que sete destes 12 sintomas neuropsiquiátricos foram observados em $50 \%$ ou mais dos pacientes com DM, quais sejam, idéias delirantes, alucinações, apatia, agitação, humor deprimido, irritabilidade e alteração do sono/vigília; ao passo que, apenas três dos 12 sintomas foram observados em $50 \%$ ou mais dos pacientes com DA, são eles agitação, humor deprimido e alteração do sono/vigília (Figura 2).

Apatia, delírios e alucinações foram significativamente mais freqüentes na DM. Idéias delirantes mostraram uma razão de chance de 3,85 e a apatia, uma razão de chance de 5,11, em favor de sua presença na DM em comparação com a DA. Quanto às alucinações, houve tendência estatística para sua maior ocorrência na DM em relação à DA (Tabela 2).

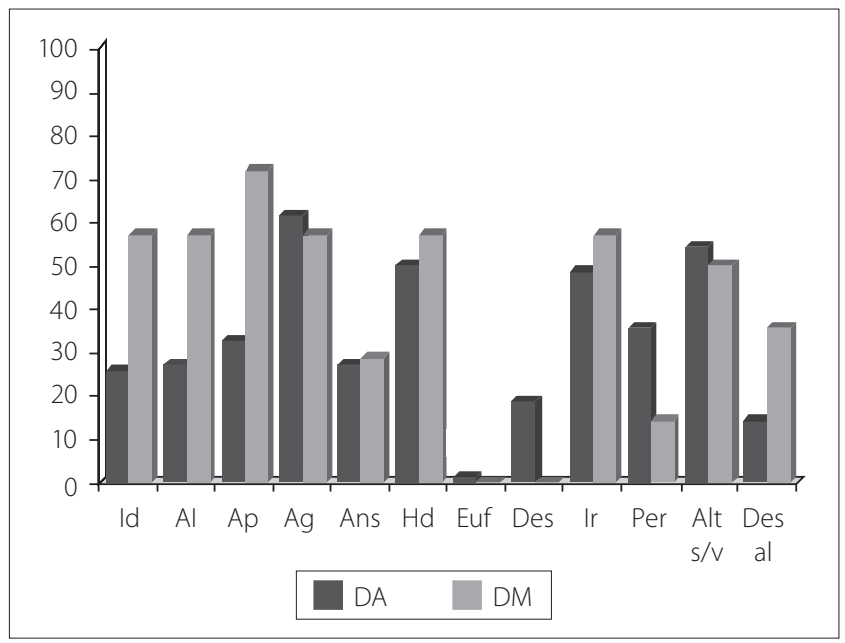

Id: idéias delirantes; Al: alucinações; Ap: apatia; Ans: ansiedade; Hd: humor deprimido; Euf: euforia; Des: desinibiçãa; Ir: irritabilidade; Per: perambulação; Alt s/v: alteração sono/vigília; Des al: desordem alimentar.

Figura 2. Percentual de alterações do comportamento em pacientes com DA e DM.

Tabela 2. Análise estatística das alterações de comportamento na DA e na DM.

\begin{tabular}{lcccccc}
\hline & $\mathrm{DA}(\mathrm{n}=\mathbf{7 0})$ & $\%$ & $\mathrm{DM}(\mathrm{n}=14)$ & $\%$ & $\mathrm{p}$ & Razão de chance (IC95\%) \\
\hline Idéias delirantes & 18 & 25,7 & 8 & 57,1 & 0,0202 & $3,85(1,03-14,84)$ \\
Alucinação & 19 & 27,1 & 8 & 57,1 & 0,0282 & $3,85(0,96-13,70)$ \\
Apatia & 23 & 32,9 & 10 & 71,7 & 0,0069 & $5,11(1,28-21,99)$ \\
Agitação & 43 & 61,4 & 8 & 57,1 & 0,7643 & $0,84(0,23-3,10)$ \\
Ansiedade & 19 & 27,1 & 4 & 28,6 & 0,9128 & $1,07(0,25-4,39)$ \\
Humor deprimido & 35 & 50 & 8 & 57,1 & 0,6254 & $1,33(0,37-4,92)$ \\
Euforia & 1 & 1,4 & 0 & 0 & 0,6527 & - \\
Desinibição & 13 & 18,6 & 0 & 0 & 0,0794 & $0(0,00-0,43)$ \\
Irritabilidade & 34 & 48,6 & 8 & 57,1 & 0,5581 & $1,41(0,39-5,21)$ \\
Perambulação & 25 & 35,7 & 2 & 14,3 & 0,1170 & $0,30(0,04-1,60)$ \\
Alteração sono /vigília & 38 & 54,3 & 7 & 50 & 0,7691 & $0,84(0,23-3,04)$ \\
Desordem alimentar & 10 & 14,3 & 5 & 35,7 & 0,059 & $3,33(0,77-14,26)$ \\
\hline
\end{tabular}

* DA como referência. 
Considerando-se a soma de sintomas relatados pelo mesmo paciente durante todo o período de seguimento, tem-se que, na DM, nove $(64,3 \%)$ pacientes tinham cinco ou mais manifestações neuropsiquiátricas, enquanto na DA, 40 $(57,1 \%)$ pacientes tinham quatro ou menos. Esta diferença, embora percentualmente fale a favor da presença de maior número de sintomas nos pacientes com DM do que naqueles com DA, não alcançou significância estatística (Tabela 3).

Em relação ao tempo de demência, 45 (64,3\%) pacientes com DA apresentavam duração de demência de quatro anos ou mais, enquanto em dez $(71,4 \%)$ pacientes com DM a duração era de três anos ou menos. Este tempo de demência nos pacientes com DM é significativamente menor do que nos pacientes com DA (Tabela 4).

Tabela 3. Número de sintomas neuropsiquiátricos em um mesmo paciente.

\begin{tabular}{lcccccc}
\hline & $\mathrm{DA}(\mathrm{n}=70)$ & $\%$ & $\mathrm{DM}(\mathrm{n}=14)$ & $\%$ & $\mathrm{p}$ & Razão de chance (IC95\%) \\
\hline$\leq 4$ sintomas & 40 & 57,1 & 5 & 35,7 & & \\
$\geq 5$ sintomas & 30 & 42,9 & 9 & 64,3 & & 2,1422 \\
\hline
\end{tabular}

Tabela 4. Tempo de demência na DA e na DM.

\begin{tabular}{ccccccc}
\hline & $\mathrm{DA}(\mathrm{n}=70)$ & $\%$ & $\mathrm{DM}(\mathrm{n}=14)$ & $\%$ & $\mathrm{p}$ & Razäo de chance $($ IC95\%) \\
\hline$\leq 3$ anos & 25 & 35,7 & 10 & 71,4 & & \\
$\leq 4$ anos & 45 & 64,3 & 4 & 28,6 & & \\
\hline
\end{tabular}

\section{DISCUSSÃO}

Os pacientes com DM apresentaram duração significativamente menor de doença se comparados aos casos com DA, sugerindo que os primeiros procuram atendimento mais precocemente. Apatia, alucinações e idéias delirantes mostraram maior freqüência na DM e, por serem sintomas compatíveis com fases avançadas de $\mathrm{DA}^{8}$, sugerem instalação mais precoce na DM. O número de alterações de comportamento em um mesmo paciente, embora seja percentualmente maior na DM, não atingiu diferença significativa entre os dois tipos de demência, talvez por limitações do tamanho amostral.

Apatia, humor deprimido e agitação figuraram entre os sintomas mais relatados em ambas as demências, o que vai ao encontro do achado prévio de estudo realizado em uma população norte-americana ${ }^{19}$. Apatia e depressão aparecem ainda entre os principais sintomas em pacientes com DA em um estudo brasileiro de base populaciona ${ }^{20}$. A relevância da apatia na DA já é bem conhecida ${ }^{13,21,22}$, sendo o principal sintoma observado em pacientes demenciados, de acordo com o que foi observado nos casos de DM do presente estudo, em que apatia foi o sintoma neuropsiquiátrico mais relatado ( $71,7 \%$ dos pacientes).
Verificou-se que $50 \%$ dos pacientes com DA e $57,1 \%$ dos pacientes com DM apresentaram queixa de humor deprimido. A alta prevalência de sintomas depressivos na DA e em demências, de maneira geral, já foi demonstrada por estudos prévios ${ }^{12,13,20}$.

Alterações do sono e vigília estavam entre os sintomas mais encontrados nos pacientes com DA com 54,3\%. Estas alterações também faziam parte dos sintomas psiquiátricos mais encontrados em pacientes com DA em estudo brasileiro, descritas em 38,3\% dos $\operatorname{casos}^{20}$.

Por sua vez, a taxa de alucinações na DA encontrada no presente estudo $(27,1 \%)$ coincide com os valores observados para demências, de modo geral, em outro estudo brasileiro ${ }^{13}$

É possível que a maior gravidade dos sintomas neuropsiquiátricos, aparecendo em maior número e de maneira mais precoce na DM, seja responsável pela procura antecipada de atendimento por estes pacientes. Considerando-se que a duração dos sintomas de demência foi obtida somandose o tempo de evolução até a primeira consulta ao tempo de seguimento do paciente no ambulatório, e que o atendimento ambulatorial esteve disponível para os dois grupos de pacientes no mesmo período, a diferença observada em relação à duração dos sintomas deveu-se ao tempo de evolução até a primeira consulta. Os pacientes com DM, provavelmente, estejam chegando no Ambulatório de Neurologia Cognitiva com menor tempo de doença ou porque o cuidador seja o responsável por levá-los a procurar por auxílio médico ou porque a presença de sintomas neuropsiquiátricos nos pacientes cause desgaste físico e emocional para este cuidador,10. Todavia, não se pode descartar a possibilidade de que evento cerebrovascular clinicamente manifesto tenha despertado mais precocemente a procura por serviço de avaliação neurológica especializada, a fim de esclarecer o declínio cognitivo.

É importante considerar que as formas de evolução das duas demências podem contribuir diretamente para a diferença do tempo de chegada dos pacientes ao serviço especializado. A DA apresenta-se inicialmente de maneira insidiosa com deterioração progressiva das funções cognitivas, porquanto alterações comportamentais, como irritabilidade, agressividade e sintomas psicóticos, usualmente aparecem nos estágios intermediário e avançado da doença. Na DM, por sua vez, a forma de apresentação mais comum é de paciente com sintomas e características clínicas típicas de DA e sinais clínicos e de neuroimagem de DCV que apresenta piora abrupta ${ }^{23}$. Espera-se, assim, que a evolução gradual na DA diante da evolução mais rápida e, eventualmente, "em degraus" na DM possibilite aos cuidadores dos primeiros pacientes tempo maior de adaptação do que aos cuidadores dos últimos, contribuindo, então, para que a demanda por ajuda seja talvez mais requisitada por cuidadores de pacientes com DM, diante dos pacientes com DA. 
As principais limitações do estudo são o pequeno tamanho amostral do grupo com DM, a natureza retrospectiva da metodologia e a falta de aplicação padronizada do inventário neuropsiquiátrico. Vale ressaltar, no entanto, que estes dois últimos vieses, ocorreram nos dois grupos de interesse.

\section{CONCLUSÕES}

Os pacientes com DM exibiram maior gravidade de sintomas neuropsiquiátricos, o que pode ter sido responsável pela busca mais precoce de assistência especializada. Os idosos que apresentam sintomas psicóticos (como alucinações e delírios), que se agitam, em especial no período noturno, que são agressivos ou, pelo contrário, totalmente apáticos, causarão em seus cuidadores sentimentos de impotência e inaptidão, fazendo que estes se sintam impelidos a buscar por ajuda qualificada. Quanto mais cedo esses sintomas se instalam, mais precoce se faz a necessidade de um profissional para orientar e intervir, buscando melhorar a qualidade de vida do idoso demenciado e de seu familiar ou cuidador. A equipe que acolhe o paciente e sua família deve estar apta a receber toda a demanda que estes trazem consigo.

Conflito de interesse: Simone Rios Fonseca e Mariana Alves de Almeida são bolsistas de iniciação científica da Fundação de Amparo à Pesquisa do Estado de Minas Gerais (Fapemig). Ana Paula Borges Santos é bolsista de iniciação científica do Conselho Nacional de Desenvolvimento Científico e Tecnológico (CNPq).

\section{REFERÊNCIAS}

1. Forlenza OV, Caramelli P, editors. Neuropsiquiatria geriátrica. São Paulo: Atheneu, 2000.

2. O'Brien JT. Vascular cognitive impairment. Am J Geriatr Psychiatry, 2006;14:724-33.

3. Herrera E, Caramelli P, Silveira AS, Nitrini R. Epidemiologic survey of dementia in a community-dwelling Brazilian population. Alzheimer Dis Assoc Disord, 2002;16:103-8.

4. Starkstein SE. Demência vascular e neuroimagem em neuropsiquiatria geriátrica. Emln: Forlenza OV, Caramelli P. Neuropsiquiatria geriátrica. Editora São Paulo: Atheneu; 1. ed, 2000. p. 221-30

5. Caramelli P, Barbosa MT. Como diagnosticar as quatro causas mais freqüentes de demência?. Rev Bras Psiquiatr. 2002; 24 (Suppl I):7-10.
6. Langa KM, Foster NL, Larson EB. Mixed dementia, emerging concepts and therapeutic implications. JAMA. 2004;292:2901-08

7. Nagy Z, Esiri MM, Jobst KA, Morris JH, King EMF, Mcdonald B, et al. The effects of additional pathology of the cognitive deficit in Alzheimer disease. J Neurophatol Exp Neurol. 1997:56:165-70.

8. Fuh JL, Wang SJ, Cummings UL. Neuropsychiatric profile in patients with Alzheimer's disease and vascular dementia. J Neurol Neurosurg Psychiatry. 2005;76:1337-41.

9. Gonzales-Salvador T, Aragano C, Lyketsos CG, Barba AC. The stress and psychological morbidity of the Alzheimer patient caregiver. Int J Geriatr Psychiatry. 1999;14:701-10.

10. Cassis SVA, Karnakis T, Moraes TA, Curiati JAE, Quadrante ACR, Magaldi RM. Correlation between burden on caregiver and clinical characteristics of patients with dementia. Rev Assoc Med Bras. 2007:53:497-501.

11. Caramelli P, Bottino CMC. Tratando os sintomas comportamentais e psicológicos da demência (SCPD). J Bras Psiquiatr. 2007;56: 83-87.

12. Almeida, OP. Sintomas psiquiátricos entre pacientes com demência atendidos em um serviço ambulatorial. Arq Neuropsiquiatr. 1999;57:937-43.

13. Vega UM, Marinho V, Engelhardt E, Laks J. Sintomas neuropsiquiátricos nas demências: relato preliminar de uma avaliação prospectiva em um ambulatório do Brasil. Arq Neuropsiquiatr. 2007;65:498-502

14. Fernández-Martínez M, Castro J, Molano A, Zarranz JJ, Rodrigo RM, Ortega R. Prevalence of neuropsychiatric symptoms in Alzheimer"s disease and vascular dementia. Curr Alzheimer Res. 2008:5:61-9.

15. American Psychiatric Association. Diagnostic and Statistical Manual of Mental Disorders. 4. ed. Washington, DC: Am Psychiatric Assoc. 1994

16. McKhann G, Drachman D, Folstein M, et al. Clinical diagnosis of Alzheimer"s disease: report of the NINCDS-ADRDA work group. Neurology. 1984;34:939-44.

17. Román GC, Tatemichi TK, Erkinjuntti T, Cummings JL, Masdeu JC, Garcia JH, et al. Vascular dementia: diagnostic criteria for research studies. Report of the NINDS-AIREN International Workshop. Neurology. 1993;43:250-60

18. Cummings JL, Mega M, Gray K, Rosenberg-Thompson S, Carusi DA, Gornbein J. The Neuropsychiatric Inventory: comprehensive assessment of psychopathology in dementia. Neurology. 1994;44:2308-14.

19. Lyketsos CG, Lopez O, Jones B, Fitzpatrick AL, Breitner J, DeKosky S. Prevalence of Neuropsychiatric Symptoms in Dementia and Mild Cognitive Impairment. JAMA. 2002;288:1475-83.

20. Tatsch MF, Bottino CMC, Azevedo D, Hototian SR, Moscoso MA, Folquitto JC, et al. Neuropsychiatric symptoms in alzheimer disease and cognitively impaired, nondemented elderly from a community-based sample in Brazil: prevalence and relationship with dementia severity. Am J Geriatr Psychiatry. 2006;14:438-45.

21. Teixeira-Jr AL, Caramelli P. Apatia na doença de Alzheimer. Rev Bras Psiquiatria. 2006;28:238-41.

22. Guimarães $H C$, Levy R, Teixeira AL, Beato RG, Caramelli P. Neurobiology of apathy in Alzheimer's disease. Arq Neuropsiquiatr. 2008;66:436-443.

23. Neto JG, Temelini MG, Forlenza OV. Diagnóstico diferencial das demências. Rev Psiquiatr Clin. 2005:32:119-130. 